\title{
APPLICATION OF DESWIRL DEVICE IN CYCLONE DUST SEPARATOR
}

\author{
M.Z. Abdullah*, Z. Husain and S.M. Fraser \\ School of Mechanical Engineering, Universiti Sains Malaysia, \\ Engineering Campus, 14300 Nibong Tebal, Penang, Malaysia
}

Received 15 November 2002, Accepted 30 March 2003

\begin{abstract}
The experimental investigations of the vortex flow inside the vortex finder (outlet duct) of the cyclone dust separator have been carried out. Preliminary study from the visualization experiment has been performed and discovered vortex instability inside the conventional vortex finder. In order to minimize the instabilities, the streamlined entry shape was inserted at the vortex finder entrance and the results showed remarkable improvement of the vortex flow instability inside the vortex finder. The velocity measurements of two main components of velocity were performed using a laser-Doppler anemometry at the cyclone vortex finder outlet. The experiments were conducted at a constant flow rate of $0.0246 \mathrm{~m}^{3} / \mathrm{s}$ with the vortex finder diameter of $64 \mathrm{~mm}$ and with several types of entrance configuration in order to improve the cyclone performance and to reduce the losses. The use of deswirl devices inside the vortex finder significantly reduced pressure drop and energy losses.
\end{abstract}

\section{INTRODUCTION}

The cyclone dust separator is a well known mechanical device for separating particles from the fluid stream. It has been used in a wide variety of industrial and manufacturing sectors such as cement, fertilizers, saw dust, plastic etc. The popularity of using the cyclone dust separator is due to the fact that it is simple and inexpensive to manufacture, compact, contains no moving parts and requires very little maintenance.

The fluid dynamics of vortex flow in the cyclone has been extensively researched for a long period. Clearly, the objectives of all cyclone separator research are to improve the efficiency and operational performance in industrial applications. Better methods of improvement are needed through a greater understanding of the physical fluid flow phenomena encountered in a conventional cyclone.

The flow pattern inside the cyclone chamber is found to be complex. The flow pattern is a combined vortex (or a Rankine vortex) with an outer vortex moving flow downward from the cyclone inlet and an inner vortex core moving flow upward into the vortex finder. The existence of this double spiral flow in high velocity cyclones have been recognised by most of the researchers (First (1949), Enoya (1956), Jirun et. al (1990), Reydon and Gauvin (1981), Zhou and Soo (1990), Fraser and Abdullah (1995, 1997)). The behaviour of the vortex flow in the

*Corresponding author-email : mezul@rocketmail.com 
cyclone is complicated by several instabilities such as vortex breakdown phenomena, recirculation zone, precessing vortex core and fluid dynamics instabilities related to the vortex shedding mechanism as widely reported in the literature (Yazdabadi et. al (1994,1994), Khater (1993)). The complexity of this motion and the existence of the secondary flows inside the chamber have so far hindered the complete understanding of the detailed mechanics of vortex flow (Dorman 1974). The three-dimensional time-dependent precessing vortex core and vortex flow oscillations are the predominant instabilities that occur inside the conventional cyclone which cause the higher energy loss and pressure drop, reduce the cyclone performance and the efficiency of particle separation. Thus, the present studies are to increase understanding of the cyclonic flow and to control the flow field parameters to establish more stable vortex structures in order to reduce the losses and pressure drop.

\section{EXPERIMENTAL SET-UP}

\subsection{The test model}

The cyclone test model was designed by Khater (1993) based on the Stairmand high efficiency design and it is made from perspex in order to accommodate the needs for LDA measurements. The detail of the test model is shown in Figure 1. It was designed with a tangential inlet duct where the duct was rectangular in cross section $(95 \mathrm{~mm}$ height $\times 38 \mathrm{~mm}$ width). At the inlet duct, the pressure tapping was made to determine the difference between static and atmospheric pressure in order to estimate the static pressure drop of the cyclone $\Delta \mathrm{P}_{\mathrm{S}}$. The static pressure drop, $\Delta \mathrm{P}_{\mathrm{S}}$ measurements across the cyclone was made using an inclined manometer. This instrument operated over the range $0-981 \mathrm{~N} / \mathrm{m}^{2}$ and could measure the pressure to an accuracy of $\pm 0.2 \%$ full scale.

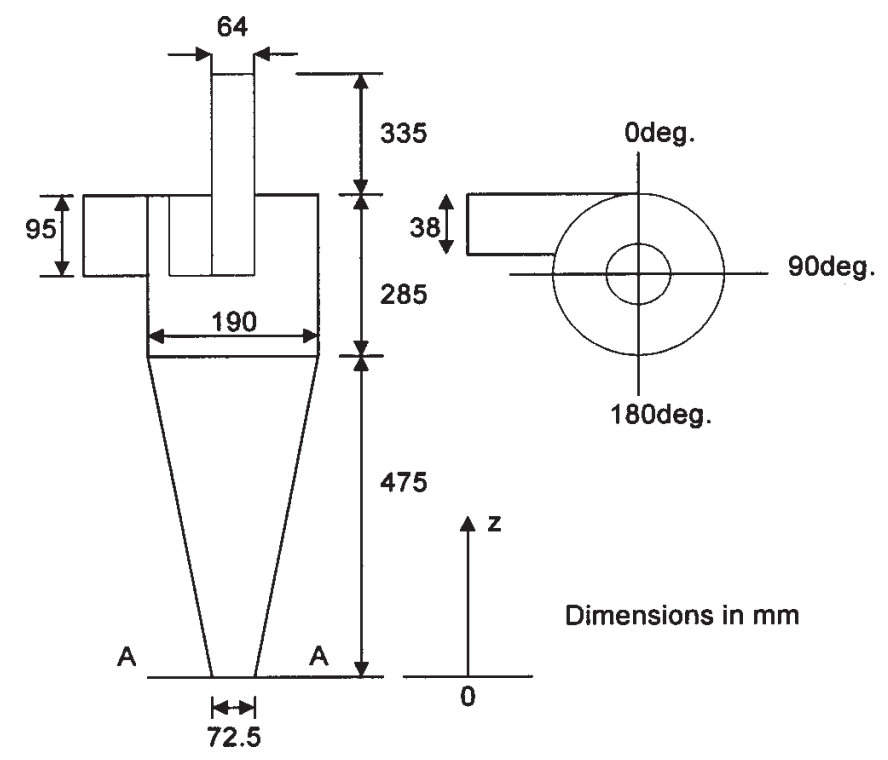

Figure 1: Model of Cylone Dust Separator. 

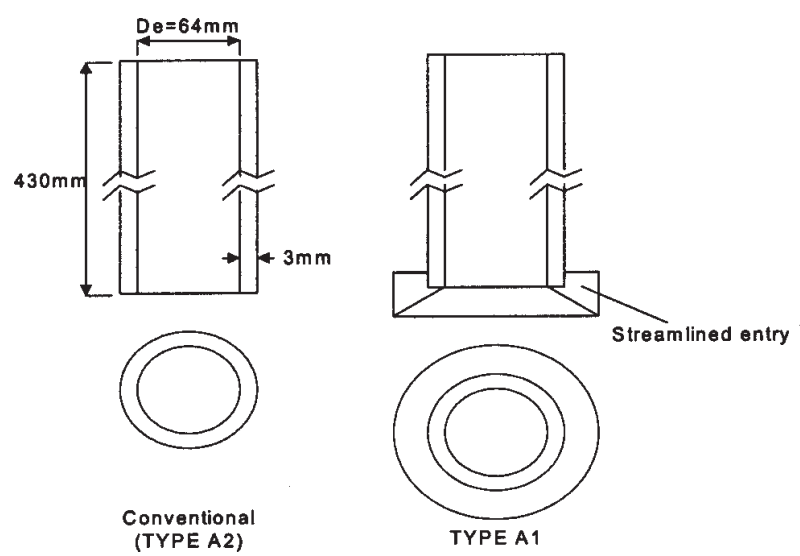

Figure 2: Conventional vortex finders without streamlined entry (Type A2) and modified vortex finder with streamlined entry (Type A 2).

The test model also consisted of the cylindrical body at the upper part with $190 \mathrm{~mm}$ ID (D) and $285 \mathrm{~mm}$ height $(\mathrm{h})$, the conical body at the lower part with inclined angle of $7^{\circ}$ and $475 \mathrm{~mm}$ height (H-h). The thickness of the test model perspex was $5 \mathrm{~mm}$ and gives good optical access for the LDA measurements. At the top part, the test model was specially constructed to allow the various diameter, $\mathrm{D}_{\mathrm{e}}$ and configuration of vortex finders used during the experimental program.

Figure 2 shows a schematic diagram of the vortex finders together with the streamlined shape at the entry, all of them are named as Type A1 and the vortex finders without the streamlined shape for conventional cyclone dust separator are called Type A2. A vortex finder diameter, $D_{e}$ of $64 \mathrm{~mm}$ and the height of $430 \mathrm{~mm}$ was used in the experimental program and made from perspex. The streamlined entries were made from aluminum and had the entry angle of $60^{\circ}$ (measured from vertical) used to improve the vortex core stability by reducing the flow disturbance at the vortex finder entrance. Figure 3 shows the diagram of the deswirl blades and deswirl device used are fitted in the bottom of the vortex finder. Three types of deswirl blade were applied in the vortex finder in order to cut the swirl velocity component. For the vortex finders with streamlined shape, its have been referred as Type B1, Type C1 and Type D1 (see Figure 3) whereas without streamlined shape they are called as Types B2, C2 and D2 respectively. Each

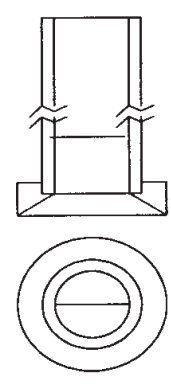

TYPE B1
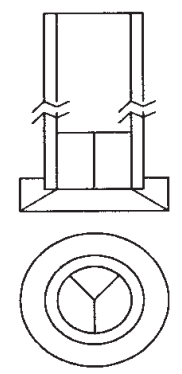

TYPE C1
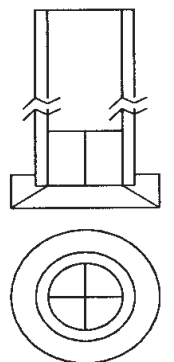

TYPE D1
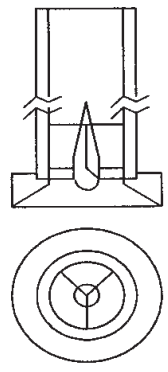

TYPE E1

Figure 3 : Various vortex finders configuration. 
blade was made from aluminum plate $130 \mathrm{~mm}$ and $0.5 \mathrm{~mm}$ thickness. The deswirl device has been designed in order to reduce the overall pressure drop and energy loss inside the vortex finder. The deswirl device (Type E1 in Figure 3) was designed from three aluminum blades and a central body. The choice of the three blades used here was based on the pressure drop results (as illustrated in Experimental Results) where the vortex finder with three blades "Type C1" produced the lowest pressure drop in the cyclone This device will be referred as Type E1 and E2 for the vortex finder with and without streamlined shape.

Air as a working fluid is drawn from ambient outside the laboratory into the reservoir chamber via a two stage axial fan which can be controlled to allow a wide range of test conditions. Ambient temperature was measured by a thermometer and ambient pressure was measured by a Barometer. It was assumed the air supplied to the cyclone test model from the ambient was perfectly isothermal.

In the reservoir chamber, the honeycomb and a wire screen were fitted in order to reduce the turbulence and to provide a uniform flow into the test section. The air was supplied to the cyclone test section through the piping system with a $100 \mathrm{~mm}$ ID. A detail construction of the reservoir with the piping system arrangement is shown in Figure 4. The air flow rate is measured by an orifice meter where it is fitted to the piping system before the air passed to the test section. Fine adjustment of the flow rate can be made using the butterfly opening gate at the reservoir chamber to bypass some of the flow.

The air flow rate was determined by an orifice plate manufactured and installed in accordance with British Standard BS 1042. It was made by Khater (1993) with a calibration factor of 0.61. The differential pressure across the orifice plate was measured (using D by D/2 tappings) using an inclined manometer. The present work was conducted at a constant flow rate of $0.0246 \mathrm{~m}^{3} / \mathrm{s}$ which was measured upstream of the position where dust was added to the air stream (Figure 4).

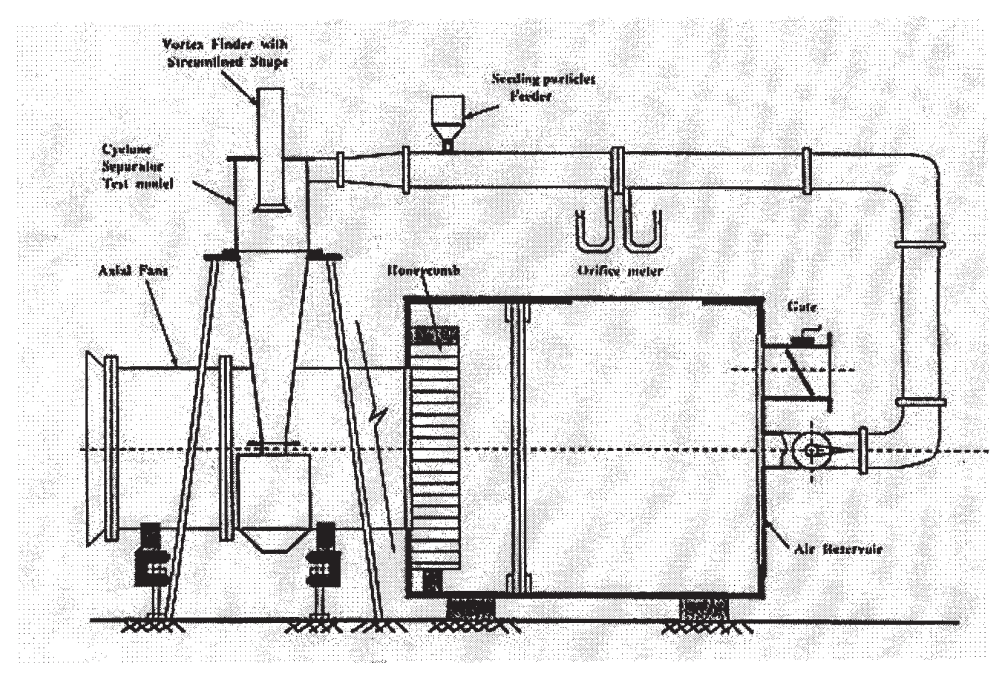

Figure 4: Air supply system 


\section{LDA equipment}

The LDA is a DANTEC two component Argon-ion laser with a fibre optic system. The Argonion laser connected to the 3-phase power supply provides a power output of 2 Watt and is operated with a water cooled system. A Bragg Cell in the transmission optics is used to apply frequency shift to the mixed color beam i.e. green $(514.5 \mathrm{~nm})$ and blue $(488 \mathrm{~nm})$. The four beams with almost equal intensity were emitted from the 2D probe and cross each other at the focal length $(600 \mathrm{~mm})$.

The backscattered light from the measuring volume is collected by the photomultiplier through the fibre optic and passes the signals to the Burst Spectrum Analysers (BSA) and oscilloscope. The data from the BSA are stored on the PC computer using the data acquisition software supplied by DANTEC Elektronik. The laser probe is mounted on a traversing mechanism allowing the movement in $\mathrm{x}$ and $\mathrm{y}$ directions. It is fully controlled by a separate PC computer via control box and the accuracy of the traversing mechanism is $0.001 \mathrm{~mm}$.

All the experimental results presented in this paper were measured at the cyclone outlet (at $\mathrm{z}=$ $1105 \mathrm{~mm}$ ) with $\mathrm{D}_{\mathrm{e}}=64 \mathrm{~mm}$, in order to study the flow structure of the vortex in the vortex finder. The measurements were made just outside at the outlet (about $10 \mathrm{~mm}$ above vortex finder), because the measurement inside the vortex finder is not possible due to the poor scattering signals obtained and high refraction of the laser beam when it is passed through the highly curved thick vortex finder wall. The velocity profiles obtained will give an indication of the flow pattern inside the vortex finder by assuming that the decay of swirl inside the vortex finder is small. Apart from the velocity profiles, this section also aimed to observe the flow instabilities such as the precessing vortex core or oscillation at the cyclone outlet and to study the effect of the streamlined entry shape on the velocity profiles.

\section{EXPERIMENTAL RESULTS}

\subsection{The flow visualization}

In the early stage of the investigation, flow visualization experiments were conducted in order to visualize the vortex core instabilities inside the vortex finder. A number of reserchers have reported vortex core instabilities such as the precessing vortex core (PVC) and vortex oscillation that occur inside the vortex finder (Yazdabadi et. al, 1995, Khater 1993). Thus, the flow visualization was performed to assist in the interpretation of the vortex core stability and to discover the causes for such problems. The flow pattern has been examined by visual and photographic observation of the powder marking on the inner surface of the vortex finder as illustrated in Figure 5. The coffee-mate powder in the ranges of 1 to $10 \mu \mathrm{m}$ is used to trace the flow inside the vortex finder.

The photographs illustrate the flow pattern inside the vortex finder without streamlined entry in the conventional cyclone as referred to Type A2 in Figure 5A and with streamlined entry (Type A1) in Figure 5B. In case of the vortex finder Type A2 (in Figure 5A), the results showed the 


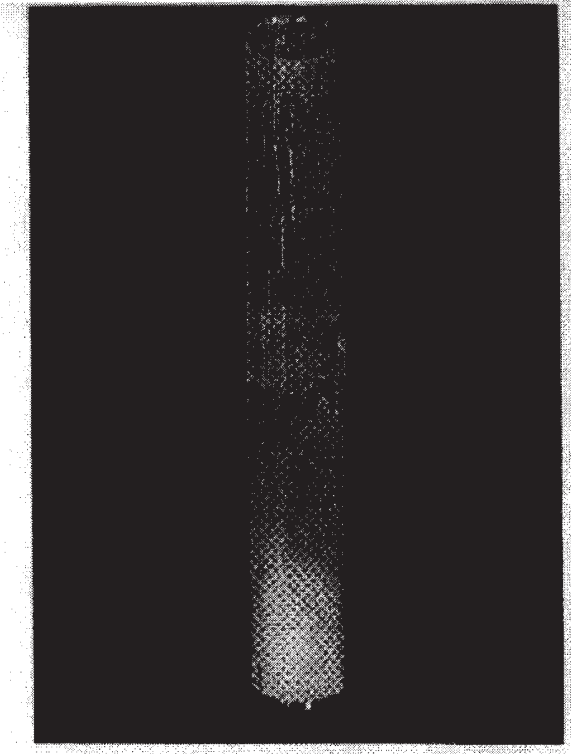

A)Vortex finder without Streamlined Entry

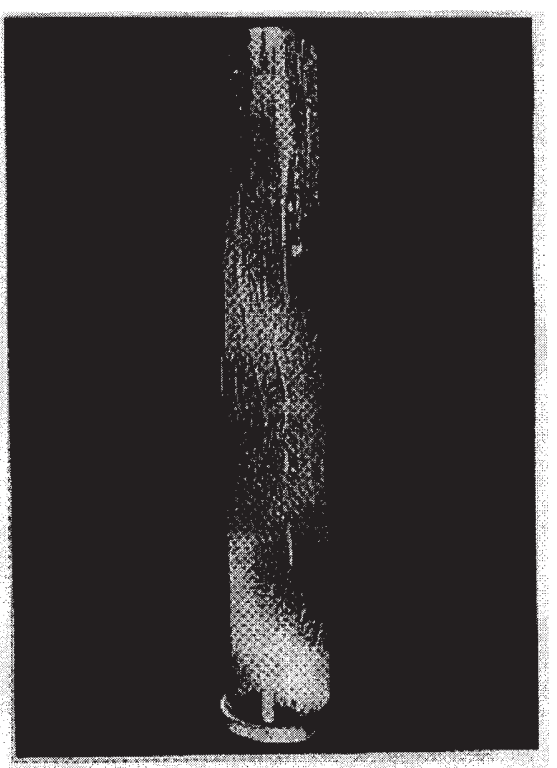

B)Vortex finder with Streamlined Entry

Figure 5 : Flow pattern inside the vortex finder without (conventional) and with streamlined entry shape

pattern of powder marking is scattered and clearly show that the vortex flow inside the vortex finder was disturbed caused by the sharp-edge and sudden contraction at the entrance region. The scattered powder marking illustrated that the overall vortex flow structure inside the vortex finder was unstable. The cause of the scattered powder is due to the generation of a precessing vortex core (PVC) and vortex flow oscillations inside the vortex finder as widely reported in the literature. The instabilities such as PVC will also generate extra losses and produce a higher pressure drop in the cyclone. In term of dust separation, the PVC will cause a pressure fluctuation and associated high levels of turbulence and mixing in the cyclone. Furthermore, the PVC is capable of exciting other resonant modes of oscillations which can vibrate dust collecting sections of the device and leads to the detriment of the dust collecting capabilities (Syred et. al (1985)).

In order to provide a stable vortex core inside the conventional vortex finder, the streamlined entry was inserted into the vortex finder entrance as shown in Figure 5B. The results show that the pattern of powder marking on the inner surface of vortex finder represents a uniform spiral without any disturbance in each vortex finder and thus, significantly improved the vortex core instability. The spiral particle markings on the inner wall of the vortex finder are clearly observed and show the upward flow angle $\theta$ is about $50^{\circ}$ with the vertical axis, and makes about 2.5 turns before the flow discharges to atmosphere. The pitch of the spiral motion is approximately equal to the internal diameter of the vortex finder, $D_{e}$ and enter approximately at $\alpha=180^{\circ}$ at the vortex finder entrance. Thus, the results show strong evidence that the streamlined-entry shape at the vortex finder entrance plays a significant role to provide a uniform flow and more stable vortex core which leads to the improvement of cyclone performance. 


\section{The swirl velocity profile}

The measured swirl velocity profiles, $u$ for different types of vortex finder configurations and $\alpha$ are shown in Figures 6 to 7 . Figures 6 and 7 illustrate the swirl velocity profiles with the vortex finder Types Al and A2 (for both with and without streamlined entry) across the angles of $180-0^{\circ}$ and $90-270^{\circ}$, to study the effect of streamlined entry to the velocity profile. The results show that the streamlined entry has no significant effect on the velocity profiles either across $180-0^{\circ}$ or $90-270^{\circ}$ and the mean velocity profiles do not show any instability although the visualization experiment illustrated the problem. The LDA equipment is not sufficient to detect such time and frequency dependent oscillations as the PVC; and should be combined with other equipment such as a pressure transducer or hot wire anemometer (Yazdabadi (1995)).

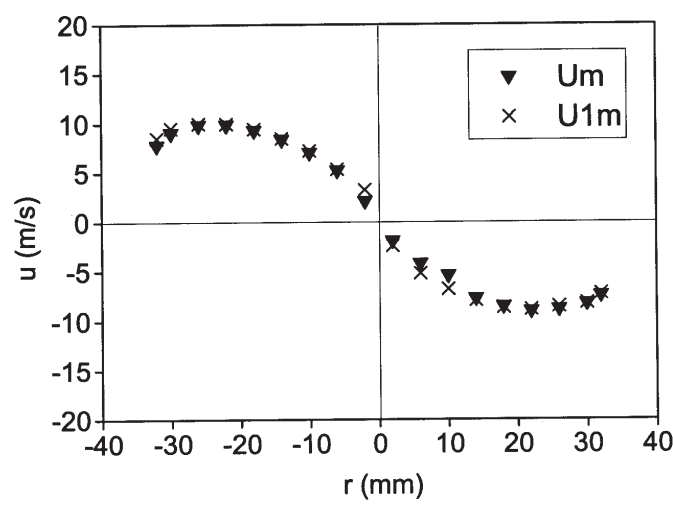

Figure 6 : Axial Velocity Profiles at outlet (across $180-0^{\circ}$ ) for vortex finder of $64 \mathrm{~mm}$ ID with and without streamlined entry shape.

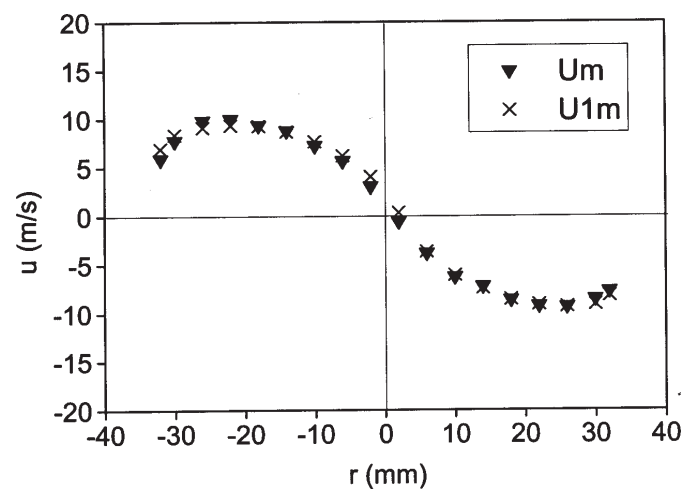

Figure 7 : Axial Velocity Profiles at outlet (across 90-270 ${ }^{\circ}$ for vortex finder of 64mm ID with and without streamlined entry shape. 
For the individual velocity profiles, it is clearly shown that the flow is rotating since the swirl velocity increases rapidly from zero at the centre. The velocity profiles can be divided into two main regions, a core region which rotates as a solid body from about $0.0 \mathrm{~m} / \mathrm{s}$ (at $\mathrm{r}=0 \mathrm{~mm}$ ) and rapidly increased up to $\mathrm{u}_{\max }$ (in the ranges of 10.5 to $16.0 \mathrm{~m} / \mathrm{s}$ ) for all figures and a free vortex region from $\mathrm{u}_{\max }$ reduces near the vortex finder wall.

The same velocity profiles at different angles are shown in Figure 10. All the profiles measured across $180-0^{\circ}, 90-270^{\circ}, 135-315^{\circ}$ and $45-225^{\circ}$ are identical. Thus, the swirl velocity profiles inside the vortex finder are axisymmetric and the swirl velocity is zero at the centre $(\mathrm{r}=0.0 \mathrm{~mm})$.

Figure 12 illustrates the swirl velocity profiles for the vortex finder Types B1, C1, D1 and E1 measured at the same position and at $\alpha=180-0^{\circ}$. Generally the results show the swirl velocity component reduces drastically in magnitude for all four types of the vortex finder configuration used compared to the results illustrated in Figures 6 and 7. The vortex finder with a single blade (Type B1) across the diameter significantly reduce the swirl velocity component to $\pm 2.5 \mathrm{~m} / \mathrm{s}$. When three blades (Type C1) are used inside the vortex finder, further reduction of swirl velocity is obtained, where the velocity in the range of -1.0 to $2.0 \mathrm{~m} / \mathrm{s}$. The result of the vortex finder with four blades (Type D1) shows that the velocity distributions are roughly similar in magnitude to those obtained by the Type $\mathrm{C} 1$ and the profile of the vortex finder with Deswirl device (a central body and guide vanes) Type E1, the swirl velocity component is almost completely removed.

From the swirl velocity profile in Figure 12, the rate of swirl energy within the vortex finder can be roughly estimated using numerical integration. The swirl energy $\Delta \mathrm{E}$ at the horizontal plane was estimated using the following equation:

$$
\Delta E=2 \pi m \int_{0}^{D_{e} / 2} u d r
$$

where the mass flow rate $m=\rho . Q$, and by assuming $\rho_{a}=1.19 \mathrm{~kg} / \mathrm{m}^{3}$ (calculated at $T_{a}=22^{\circ}$ and $\mathrm{P}_{\mathrm{a}}=758 \mathrm{mmHg}$.), thus $\mathrm{m}=0.0293 \mathrm{~kg} / \mathrm{s}$, and $\mathrm{u}$ is the swirl velocity distribution across the outlet. A polynomial equation was fitted to the velocity distribution of $\mathrm{u}$ value with $\mathrm{r}(\mathrm{u}=\mathrm{f}(\mathrm{r}))$ by using curve fitting software, thus the estimation of the rate of swirl energy $\Delta \mathrm{E}$ inside the vortex finder (Type A1) is found to be $43.0 \chi 10^{-3} \mathrm{~J}$ whereas the rate of swirl energy obtained inside the vortex finder Type E1 is very much lower $\left(1.65 \chi 10^{-3} \mathrm{~J}\right)$ and reduced about $96 \%$ compared to the conventional cyclone. Thus, from these results, one can conclude that the vortex finder with Deswirl device produces lower energy loss compared to the conventional type of cyclone.

\section{The axial velocity profile}

The axial velocity profiles, $w$ are shown in Figures 8 to 9 . They are measured across $\alpha=$ $180-0^{\circ}$ and for the vortex finder Types $\mathrm{Al}$ and A2. Generally, the profiles obtained show the same trend indicating a ' $\mathrm{V}$ ' shape, the velocity is higher close to the wall and drops sharply to the centre. The velocity distributions recorded for the two different vortex finder configurations 
(Types Al and A2) do not show any significant changes in shape, since both of the profiles are almost identical.

For individual profiles, the highest magnitude is found close to the wall roughly in the range of 12 to $13 \mathrm{~m} / \mathrm{s}$. A lower magnitude is located at the axis in the range 0 to $-2.0 \mathrm{~m} / \mathrm{s}$. The negative signs show that the velocity is directed downward into the vortex finder due to reversed flow at this region (called the recirculation zone) and almost at the centre. The results clearly show that the mean flow is axis-symmetric. The pressure at this region is lower than atmospheric, causing some air to be sucked back into the outlet duct and leads to a negative axial velocity component.

In Figures 11 and 13, all the types of vortex finder configurations are plotted on the same graph in order to observe the difference in velocity distributions more easily. The profiles show a

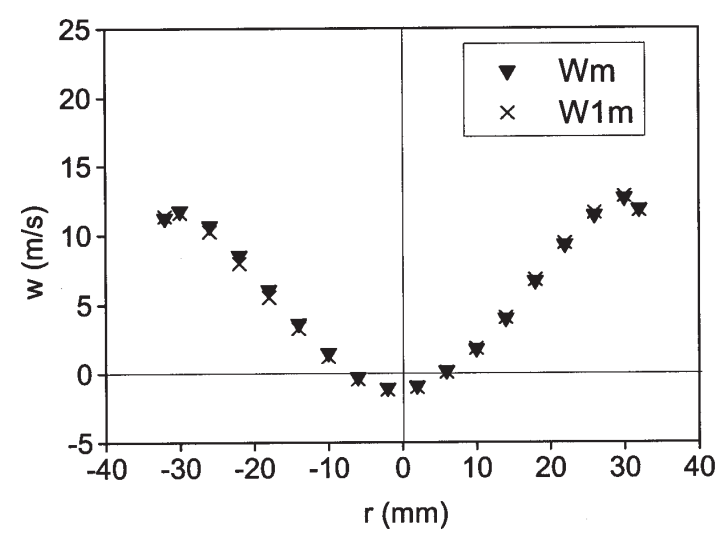

Figure 8 : Axial Velocity Profiles at outlet (across $180-0^{\circ}$ ) for vortex finder of $64 \mathrm{~mm}$ ID with and without streamlined entry shape.

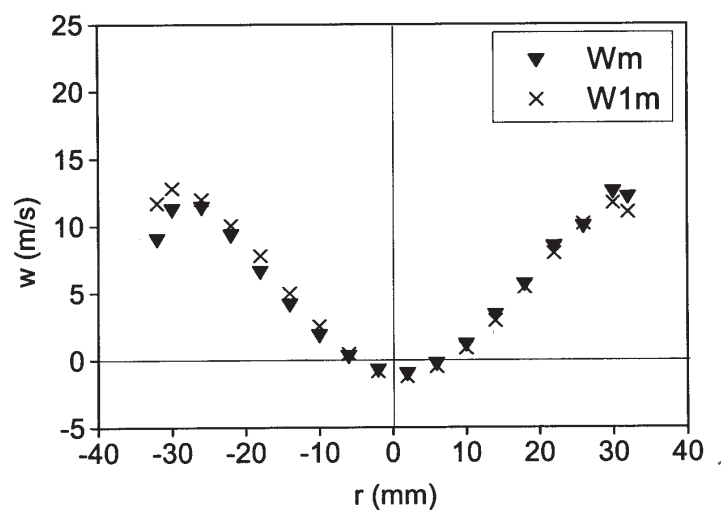

Figure 9 : Axial Velocity Profiles at outlet (across 90-270 ${ }^{\circ}$ ) for vortex finder of 64mm ID with and without streamlined entry shape. 


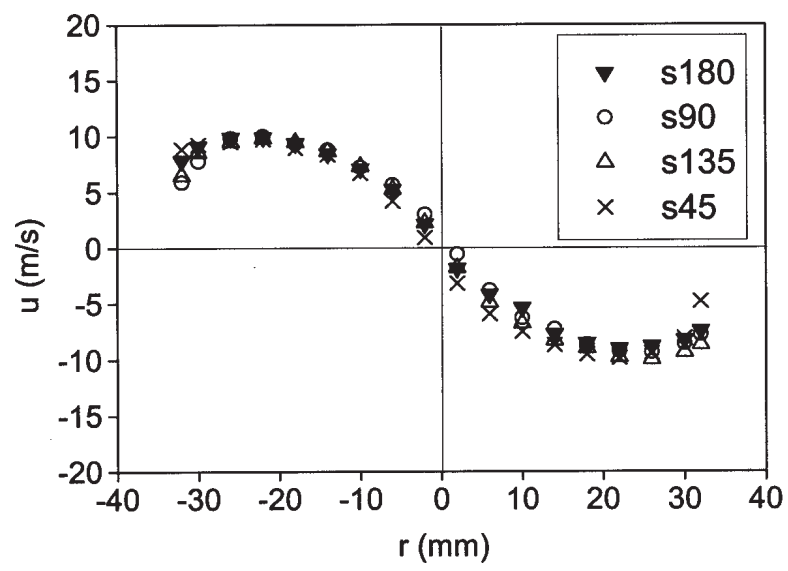

Figure 10 : Swirl Velocity Profiles at outlet (across 180-0,90-270, 135-315 and 45-225 ) for vortex finder of $64 \mathrm{~mm} I D$.

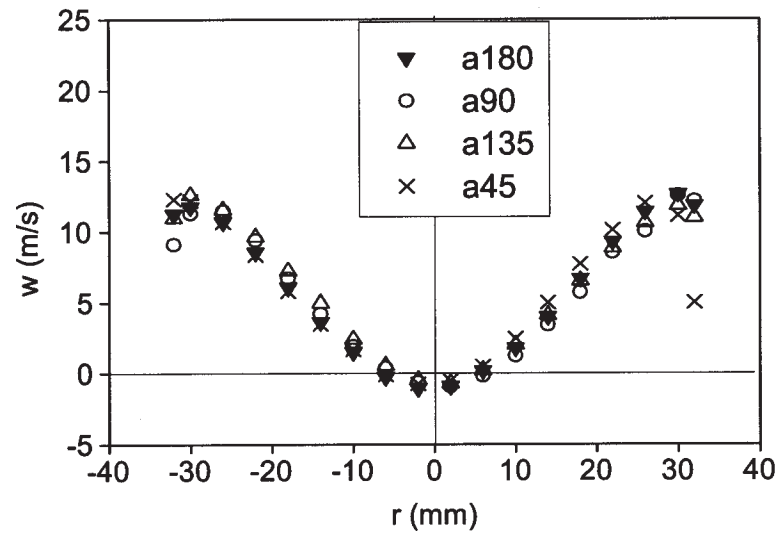

Figure 11 : Axial Velocity Profiles at outlet (across 180-0,90-270, 135-315 and 45-225 ${ }^{\circ}$ ) for vortex finder of $64 \mathrm{~mm} I D$.

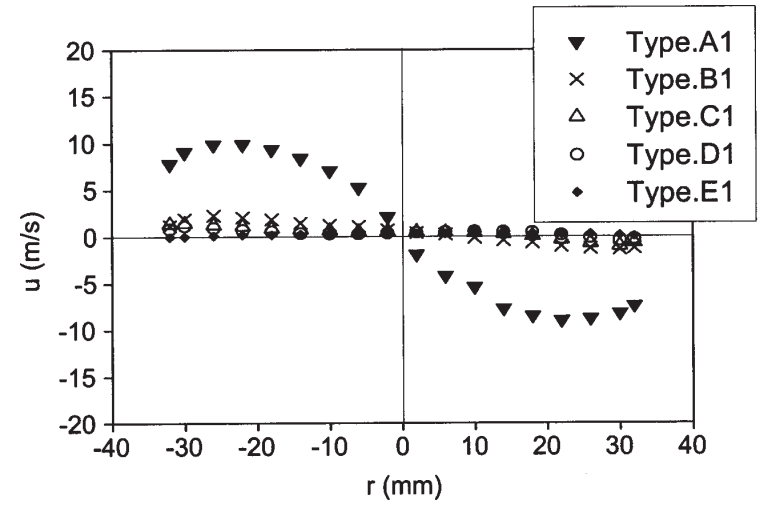

Figure 12 : Swirl Velocity Profiles at outlet (across $180-0^{\circ}$ ) and for vortex finder of 64mm ID. 


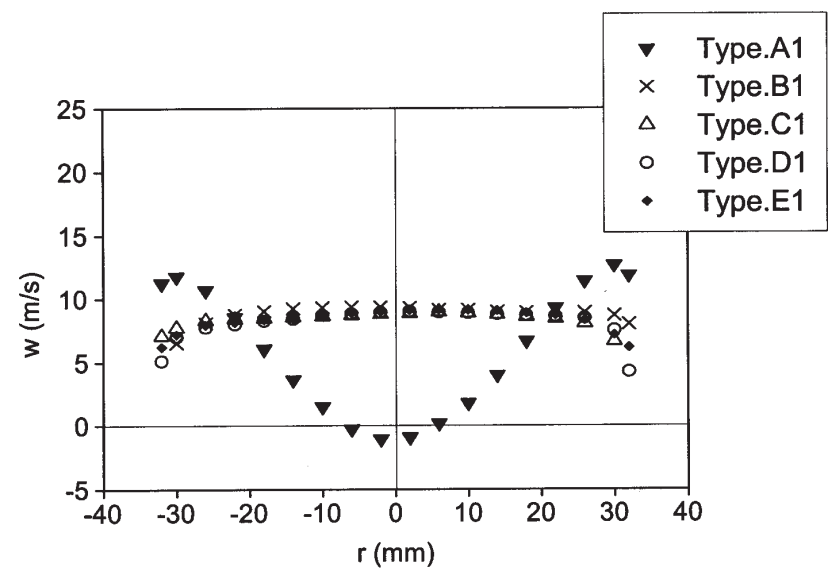

Figure 13 : Axial Velocity Profiles at outlet (across 180-0 $0^{\circ}$ ) and for vortex finder of 64mm ID.

dramatic change compared to the axial velocity distribution without the deswirl blade (Type A1). Clearly the modified vortex finder entrance (Type B1, C1, D1 and E1) has a significant effect on the velocity profile and has eliminated the reverse flow zone as reported by Yazdabadi (1995). Generally, Types B, C, D and E show insignificant change in their axial velocity profiles relative to each other.

\section{Second moment of velocity}

From the LDA velocity measurements the variations of the turbulent kinetic energy $\mathrm{k}$ as:

$$
k=\frac{1}{2}\left({\overline{u^{\prime}}}^{2}+{\overline{w^{\prime}}}^{2}+{\overline{v^{\prime}}}^{2}\right)
$$

for different vortex finder entrance configuration can be observed in Figures 14 to 15.

The swirl component of turbulent kinetic energy ${\overline{w^{\prime}}}^{2}$ profile for Type Al Figure 14 shows the peak (about $28 \mathrm{~m}^{2} / \mathrm{s}^{2}$ ) at the axis decreases rapidly towards the wall with a small increase at the wall region, whereas with the modified vortex finder configuration (Types Al, B1, C1, D1 and E1) shows that the swirl component of turbulent kinetic energy $\bar{u}^{\prime}$ is small and almost constant across the outlet (except at the wall region) roughly in the range of 0.0 to $3.0 \mathrm{~m}^{2} / \mathrm{s}^{2}$ with up to $97 \%$ reduction compared with $\mathrm{Al}$. The profiles also show that the modification of vortex finder entrance with Types $\mathrm{C} 1, \mathrm{D} 1$ and E1 produced the lowest values of ${\overline{u^{\prime}}}^{2}$.

The axial component of turbulent energy ${\overline{w^{\prime}}}^{2}$ in Figure 15 shows a similar trend for the vortex finder Types B1, C1, D1 and E1 compared to Type Al, i.e. the peak and peak magnitude do not occur at the centre and the magnitude is reduced significantly and roughly up to $90 \%$ reduction compared to Type A1. For the individual profiles, again $\overline{u^{\prime}}$ are uniformly distributed across the outlet with a small increase close the wall. Again, these results proved that the use of the Deswirl blades or Deswirl device significantly reduced the turbulent kinetic energy. 


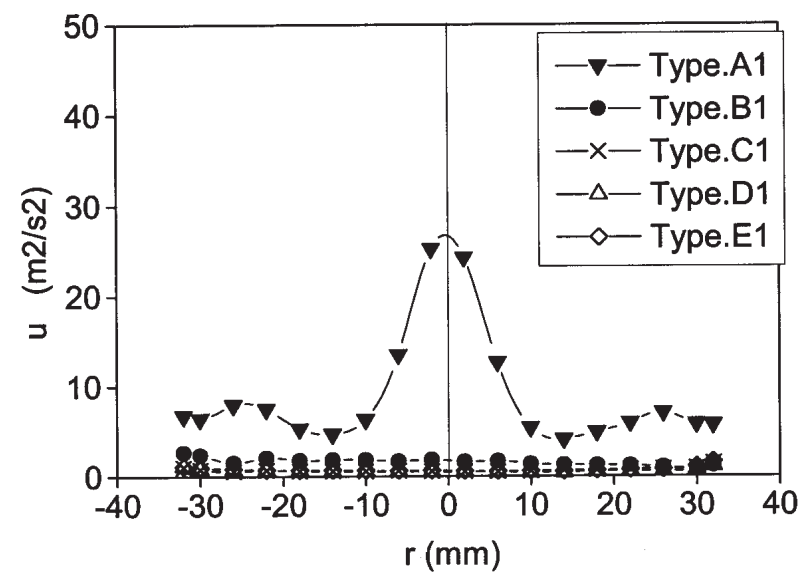

Figure 14 : Swirl component of turbulent kinetic energy at outlet (across 180-0 $0^{\circ}$ and for vortex finder of $64 \mathrm{~mm}$ ID.

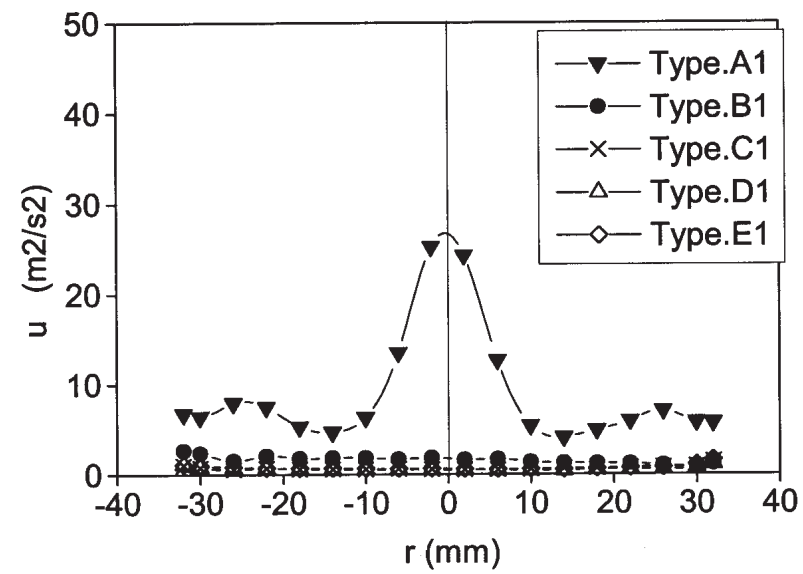

Figure 15 : Axial component of turbulent kinetic energy at outlet (across 180- $0^{\circ}$ ) and for vortex finder of $64 \mathrm{~mm}$ ID.

\section{Pressure drop}

The measurement of the pressure drop in the cyclone is one of the methods used to justify the cyclone performance where the lower pressure drop also will give the lower energy loss. Thus, the present study is aimed at finding a solution in order to reduce the pressure drop in the cyclone by modifying the vortex finder entrance configurations and to identify the best vortex finder configuration.

The pressure drop is defined as a combined static and dynamic pressure as:

$$
\Delta P=\Delta P_{S}+\rho \frac{U_{i n}^{2}}{2}
$$


where

$\triangle \mathrm{P}_{\mathrm{S}}=$ static pressure difference from cyclone inlet to outlet

$\mathrm{U}_{\text {in }}=$ average inlet velocity $(=7.28 \mathrm{~m} / \mathrm{s}$ measured using LDA $)$

$\rho=$ air density (assumed $1.19 \mathrm{~kg} / \mathrm{s}$ at the ambient temperature of $22^{\circ}$ and pressure of 758 mmHg.)

and the pressure drop coefficient or Euler number $\xi$ is defined as

$$
\xi=\frac{\Delta P}{\frac{1}{2} \rho U_{i n}}
$$

All the results discussed here are based on the coefficient pressure drop or Euler number $\xi$. The pressure drop across the cyclone was measured by tapping pressure at the inlet duct against the atmospheric. Although a number of factors could influence the pressure drop, only a few factors which related to the effect of the modified vortex finder configuration will be discussed in this paper.

The effect of the streamlined shape at the vortex finder entrance on the pressure drop is shown in Table 1. The results indicate that the use of streamlined shape at the vortex finder entrance (Type A1) reduces the pressure drop as much as 5\% compared to the conventional vortex finder (Type A2). From the results one can conclude that the sudden contraction incurred at the vortex finder with a sharp-edge will increase the cyclone pressure drop, thus better methods such as the application of streamlined entry significantly reduced the pressure drop.

A number of tests have been carried with the different type of vortex finder configurations. All five different types of vortex finder configurations are plotted on the same graph in order to compare them more easily, and the comparisons are made relative to the pressure drop in the cyclone without vortex finder modification (Type A2). Generally, all six graphs show similar trends where the smaller $\mathrm{D}_{\mathrm{e}}$ produces higher pressure drop and for the bigger $\mathrm{D}_{\mathrm{e}}$ produces lower pressure drop. From the results, the lowest pressure drop is obtained for the vortex finder Type El (using the Deswirl device), the reduction is up to $19 \%$. The results also show the use of

Table 1 : The Variation of Euler number $\xi$ with the different vortex finder configurations

\begin{tabular}{|c|c|c|}
\hline Туре & $\begin{array}{c}\text { Vortex Finder with } \\
\text { Streamlined Entry Shape }\end{array}$ & $\begin{array}{l}\text { Vortex Finder without } \\
\text { Streamlined Entry Shape }\end{array}$ \\
\hline A & 11.0 & $\begin{array}{c}11.5 \\
\text { (Conventional Cyclone) }\end{array}$ \\
\hline B & 10.7 & 11.3 \\
\hline $\mathrm{C}$ & 10.7 & 11.2 \\
\hline $\mathrm{D}$ & 10.8 & 11.2 \\
\hline $\mathrm{E}$ & 9.3 & 10.0 \\
\hline
\end{tabular}


Deswirl blades with streamlined shape i.e. Types B1, C1 and D1; the vortex finder with three deswirl blades has a lower pressure drop than a single or four deswirl blade configuration of reduction up to $7 \%$. Based upon the results, three vanes were chosen for the deswirl device Type E1 (see Figure 3) in the present study.

\section{CONCLUSIONS}

The flow instability generated from the sudden contraction of the vortex flow at the sharp-edge of vortex finder entrance has been observed using the dust marking on the inner surface of the vortex finder. The streamlined shape was introduced at the vortex finder entrance, the results illustrated the improved the vortex core stability and significantly reduced the pressure drop.

The use of the deswirl blades inside the vortex finder successfully cut the swirl velocity and partially converted the swirl energy back to the pressure energy and reduced the pressure drop. The highest reduction in pressure drop was obtained with three deswirl blades. The proper design of Deswirl device (a central body with three guide vanes) provides a uniform axial velocity profile, further reduction in the swirl velocity and, significantly reduced turbulent kinetic energy which gives a further reduction in pressure drop.

\section{REFERENCES}

1. Dorman, R.G., (1974), Dust Control an Air Cleaning, Pergamon, pp. 236-279.

2. First, M.W., (1949), ASME Annual General Meeting, no. 49A-127, pp. 1-25.

3. Fraser, S.M. and Abdullah, M.Z., (1995), Laser Anemometry ASME, FED-vol. 229, pp. 395-403.

4. Fraser, S.M., Abdel Razek, A.M. and Abdullah, M.Z. (1997), Proc. Instn. Mech. Engrs, vol. 211, Part E, pp. 247-257.

5. Enoya, K., (1956), Memoirs of the Faculty of Engineering, Nagoya University, vol. 5, pp. 131-178.

6. Jirun, X., Qian, L. and Jicun, Q. (1990), Filtration and Separation, pp. 276-278 (July/ August).

7. Khater, R.M.H. (1993), Laser Anemometry and Computer Simulation of Swirling Flows in a Model Cyclone Separator, Ph.D. Thesis, Zagazig University, Egypt.

8. Reydon, R.F. and Gauvin, W.H. (1981), The Canadian Journal of Chemical Engineering, vol. 59 (February)

9. Syred, N., Biffin, M. and Wright, M. (1985), Filtration and Separation, pp. 367-370 (November/December).

10. Yazdabadi, P.A. (1995), A Study of the Precessing Vortex Core in a Cyclone Dust Separator and a Method of Prevention, Ph.D Thesis, University of Wales, Cardiff.

11. Yazdabadi, P.A., Griffiths, A.J. and Syred, N. (1994), Experiments in Fluid, vol. 17, p. 8495.

12. Yazdabadi, P. A., Griffiths, A.J. and Syred, N. (1994), Proc. Instn. Mech. Engrs, vol. 208, pp. 147-154.

13. Zhou, L.X. and Soo, S.L. (1990), Powder Technology, vol. 63, pp. 45-53. 